\title{
Energy-Momentum Problem of Bell-Szekeres Metric in General Relativity and Teleparallel Gravity
}

\author{
M. Sharif and Kanwal Nazir \\ Department of Mathematics, University of the Punjab, \\ Quaid-e-Azam Campus, Lahore-54590, Pakistan
}

Received on 4 December, 2007

\begin{abstract}
This paper is devoted to the investigation of the energy-momentum problem in two theories, i.e., General Relativity and teleparallel gravity. We use Einstein, Landau-Lifshitz, Bergmann-Thomson and Möller's prescriptions to evaluate energy-momentum distribution of Bell-Szekeres metric in both the theories. It is shown that these prescriptions give the same energy-momentum density components in both General Relativity and teleparallel theory. Möller's prescription yields constant energy in both the theories.
\end{abstract}

Keywords: Energy-Momentum; Bell-Szekeres Metric

\section{INTRODUCTION}

One of the most interesting problems which remains unsolved, since the birth of General Theory of Relativity (GR), is the energy-momentum localization. To find a generally accepted expression, there have been different attempts. However, some attempts to define the energy-momentum density for the gravitational field lead to prescriptions that are not true tensors. The first of such attempts was made by Einstein [1] himself who proposed an expression for the energymomentum distribution of the gravitational field. Following Einstein, many scientists like Landau-Lifshitz [2], Papapetrou [3], Bergmann-Thomson [4] and Möller [5] introduced their own energy-momentum complexes. All these prescriptions, except Möller, are restricted to do calculations only in Cartesian coordinates.

The notion of energy-momentum complexes was severely criticized for a number of reasons. Firstly, the nature of a symmetric and locally conserved object is non-tensorial and thus its physical interpretation appeared obscure [6]. Secondly, different energy-momentum complexes could yield different energy-momentum distributions for the same gravitational background [7,8]. Finally, energy-momentum complexes were local objects while it was usually believed that the suitable energy-momentum of the gravitational field cannot be localized [9]. An alternate concept of energy, called quasi-local energy, was developed by Penrose and many others [10,11]. Although, these quasi-local masses are conceptually very important, yet these definitions have serious problems. Chang et al. [12] showed that energy-momentum complexes are actually quasi-local and legitimate expressions for the energy-momentum.

Virbhadra [13-15] and his collaborator [16] showed that different energy-momentum complexes yield the same results for a general non-static spherically symmetric metric of the KerrSchild class. These definitions comply with the quasi-local mass definition of Penrose for a general non-static spherically symmetric metric of the Kerr-Schild class. However, these prescriptions disagree in the case of the most general nonstatic spherically symmetric metric. Aguirregabiria et al. [17] proved the consistency of the results using different energymomentum complexes for any Kerr-Schild class metric. Xulu
$[18,19]$ extended this investigation and found the same energy distribution in the Melvin magnetic and Bianchi type I universes. Chamorro and Virbhadra [20] and Xulu [21] studied the energy distribution of the charged black holes with a dilaton field. Ramdinschi and Yang [22], Vagenas [23], Gad [24] and Xulu [25] investigated the energy distribution of the string black holes using different prescriptions. Using Einstein and Landau-Lifshitz complexes, Cooperstock [26] and Rosen [27] found that total energy of the closed Friedmann-RobertsonWalker (FRW) spacetime vanishes everywhere. With the help of Einstein energy-momentum complex, Banerjee and Sen [28] found that the total energy density of Bianchi type-I universe is zero everywhere. However, there exist examples [2932] which do not support this viewpoint.

It has been argued $[33,34]$ that the energy-momentum problem can also be localized in the framework of the teleparallel theory (TPT) of gravity. This theory has been considered long time ago in connection with attempts to define the energy of the gravitational field. Möller [35] was probably the first to notice that the tetrad description of the gravitational field allows a more satisfactory treatment of the gravitational energy-momentum than does GR. Using the teleparallel version of Einstein and Landau-Lifshitz complexes, Vargas [36] found that total energy of the closed FRW spacetimes vanishes everywhere. This result agrees with the previous work of Cooperstock [26] and Rosen [27]. It has been shown [37] that the results of Bianchi types I and II in TPT are consistent with the results in GR. Recently, Salti et al. [38,39] have calculated energy-momentum densities for some particular spacetimes by using different prescriptions both in GR and TPT and found the same results. However, there exist some spacetimes that do not provide consistent results both in GR and TPT. Sharif and Jamil [40] considered Lewis-Papapetrou metric and found that the results in TPT do not coincide with those obtained in GR [41].

This paper explores Bell-Szekeres metric by evaluating its energy-momentum distribution using different prescriptions both in GR and TPT. We would like to present results rather giving the details as these are available in the literature [40,41]. The paper has been organized as follows: Section 2 is devoted to present the prescriptions of the Einstein, LandauLifshitz, Bergmann-Thomson and Möller energy-momentum 
both in GR and TPT. In section 3, we find the energymomentum distribution of Bell-Szekeres metric both in GR and TPT using these prescriptions. Finally, section 4 provides a summary and discussion of the results obtained.

\section{ENERGY-MOMENTUM COMPLEXES}

In this section, we shall briefly outline the Einstein, Landau-Lifshitz, Bergmann-Thomson and Möller prescriptions used to calculate energy-momentum distribution of a metric both in GR and TPT.

\section{A. Energy-Momentum Complexes in GR}

For Einstein prescription, the energy-momentum density is given in the form $[5,42]$

$$
\Theta_{a}^{b}=\frac{1}{16 \pi} H_{a, c}^{b c}, \quad(a, b, c=0,1,2,3),
$$

where $H_{a}^{b c}=-H_{a}^{c b}$ is given by

$$
H_{a}^{b c}=\frac{g_{a d}}{\sqrt{-g}}\left[-g\left(g^{b d} g^{c e}-g^{c d} g^{b e}\right)\right]_{, e} .
$$

Here $g$ is the determinant of the metric tensor $g_{\mu \nu}$ and comma denotes ordinary differentiation. Notice that $\Theta_{0}^{0}$ is the energy density, $\Theta_{i}^{0}(i=1,2,3)$ are the momentum density components and $\Theta_{0}^{i}$ are the energy current density components. The momentum four-vector is defined as

$$
p_{a}=\iint_{V} \int \Theta_{a}^{0} d x^{1} d x^{2} d x^{3}
$$

where $p_{0}$ gives the energy and $p_{1}, p_{2}$ and $p_{3}$ are the momentum components while the integration is taken over the hypersurface element described by $t=$ constant . Einstein's conservation law becomes

$$
\frac{\partial \Theta_{a}^{b}}{\partial x^{a}}=0
$$

Landau-Lifshitz energy-momentum density can be given in the form [2]

$$
L^{a b}=\frac{1}{16 \pi} \ell_{, c d}^{a b c d}
$$

where

$$
\ell^{a b c d}=(-g)\left(g^{a b} g^{c d}-g^{a c} g^{b d}\right) .
$$

The quantities $L^{00}$ and $L^{0 i}$ represent the energy density and the total momentum (energy current) densities respectively.

The energy-momentum prescription of BergmannThomson is given by [4]

$$
B^{a b}=\frac{1}{16 \pi} M_{, c}^{a b c},
$$

where

$$
M^{a b c}=g^{a d} V_{d}^{b c}
$$

with

$$
V_{d}^{b c}=\frac{g_{d e}}{\sqrt{-g}}\left[-g\left(g^{b e} g^{c f}-g^{c e} g^{b f}\right)\right]_{, f}
$$

The quantities $B^{00}$ and $B^{i 0}$ represent energy and momentum densities respectively. The Bergmann-Thomson energymomentum satisfies the following local conservation law

$$
\frac{\partial B^{a b}}{\partial x^{b}}=0
$$

in any coordinate system. The energy-momentum components are given by

$$
p^{a}=\iint_{V} \int B^{a 0} d x^{1} d x^{2} d x^{3} .
$$

Using Gauss theorem, the above integral takes the form

$$
p_{a}=\frac{1}{16 \pi} \iint H_{a}^{0 b} n_{b} d S
$$

where $n_{b}$ is the outward unit normal vector to an infinitesimal surface element $d S$. The quantities $p_{i}$ give momentum components while $p_{0}$ gives the energy.

All the energy-momentum complexes mentioned above are coordinate dependent and give meaningful results only when the calculations are carried out in Cartesian coordinates. To overcome this deficiency, Möller [5] introduced another energy-momentum pseudo-tensor $M_{a}^{b}$ which is coordinate independent given as

$$
M_{a}^{b}=\frac{1}{8 \pi} K_{a, c}^{b c},
$$

where 


$$
K_{a}^{b c}=\sqrt{-g}\left(g_{a d, e}-g_{a e, d}\right) g^{b e} g^{c d},
$$

and $K_{a}^{b c}$ is antisymmetric in its upper indices. This satisfies the conservation law

$$
\frac{\partial M_{a}^{b}}{\partial x^{b}}=0
$$

where $M_{0}^{0}$ is the energy density, $M_{i}^{0}$ are momentum density components and $M_{0}^{i}$ are the components of energy current density.

\section{B. Energy-Momentum Complexes in TPT}

The teleparallel version of Einstein, Bergmann-Thomsan and Landau-Lifshitz energy-momentum complexes are given [36], respectively, as

$$
\begin{gathered}
h E_{\rho}^{\mu}=\frac{1}{4 \pi} U_{\rho}^{\mu \nu}{ }_{, v}, \\
h B^{\mu \rho}=\frac{1}{4 \pi}\left[g^{\mu \theta} U_{\theta}^{\rho \nu}{ }_{, v}\right], \\
h L^{\mu \rho}=\frac{1}{4 \pi}\left[h g^{\mu \theta} U_{\theta}{ }^{\rho \nu}{ }_{, v}\right],
\end{gathered}
$$

where $h=\operatorname{det}\left(h_{\mu}^{a}\right)$ and $U_{\rho}{ }^{\mu v}$ is the Freud's superpotential given as

$$
U_{\rho}^{\mu v}=h S_{\rho}^{\mu v}
$$

Here $S^{\rho \mu v}$ is the tensor

$$
\begin{aligned}
S^{\rho \mu \nu}= & m_{1} T^{\rho \mu \nu}+\frac{m_{2}}{2}\left(T^{\mu \rho v}-T^{v \rho \mu}\right)+ \\
& \frac{m_{3}}{2}\left(g^{\rho v} T_{\theta}^{\theta \mu}-g^{\rho \mu} T_{\theta}^{\theta v}\right]
\end{aligned}
$$

with $m_{1}, m_{2}$ and $m_{3}$ as the three dimensionless coupling constants of the teleparallel gravity. For the teleparallel equivalent of GR, the specific choice of these three constants are

$$
m_{1}=\frac{1}{4}, \quad m_{2}=\frac{1}{2}, \quad m_{3}=-1 .
$$

To calculate this tensor, we evaluate Weitzenböck connection [43]

$$
\Gamma_{\mu \nu}^{\theta}=h_{a}^{\theta} \partial_{\nu} h_{\mu}^{a}
$$

which is used to find the corresponding torsion [44]

$$
T_{\mu v}^{\theta}=\Gamma_{v \mu}^{\theta}-\Gamma_{\mu v}^{\theta} .
$$

Thus the momentum four-vector for Einstein, BergmannThomsan and Landau-Lifshitz energy-momentum complexes will be

$$
\begin{aligned}
& p_{\mu}^{E}=\int_{\Sigma} h E_{\mu}^{0} d x d y d z, \\
& p_{\mu}^{B}=\int_{\Sigma} h B_{\mu}^{0} d x d y d z, \\
& p_{\mu}^{L}=\int_{\Sigma} h L_{\mu}^{0} d x d y d z .
\end{aligned}
$$

Now we discuss Möller energy-momentum complex in the context of TPT. Mikhail et al. [33] defined the superpotential (which is antisymmetric in its last two indices) of the Möller tetrad theory as

$$
\begin{gathered}
U_{\mu}^{\vee \beta}=\frac{\sqrt{-g}}{2 \kappa} P_{\chi \rho \sigma}^{\tau \vee \beta}\left[\phi^{\rho} g^{\sigma \chi} g_{\mu \tau}-\right. \\
\left.\lambda g_{\tau \mu} K^{\chi \rho \sigma}-g_{\tau \mu}(1-2 \lambda) K^{\sigma \rho \chi}\right],
\end{gathered}
$$

where

$$
P_{\chi \rho \sigma}^{\tau \nu \beta}=\delta_{\chi}^{\tau} g_{\rho \sigma}^{\nu \beta}+\delta_{\rho}^{\tau} g_{\sigma \chi}^{\nu \beta}-\delta_{\sigma}^{\tau} g_{\chi \rho}^{\nu \beta}
$$

and $g_{\rho \sigma}^{v \beta}$ is a tensor quantity defined by

$$
g_{\rho \sigma}^{v \beta}=\delta_{\rho}^{v} \delta_{\sigma}^{\beta}-\delta_{\sigma}^{v} \delta_{\rho}^{\beta}
$$

Here $K^{\sigma \rho \chi}$ is a contortion tensor, $\lambda$ is a free dimensionless coupling constant of TPT, $\kappa$ is the coupling constant and $\phi_{\mu}$ is the basis vector field given by

$$
\phi_{\mu}=T_{v \mu}^{v}
$$

The energy-momentum density is defined as

$$
M_{\mu}^{v}=U_{\mu}{ }^{v \rho},
$$

The energy $E$ contained in a sphere of radius $R$ is expressed by the volume integral as

$$
p_{\mu}(R)=\int_{r=R} \iint U_{\mu}^{0 \rho}{ }_{, \rho} d x^{3},
$$

$$
p_{\mu}(R)=\int_{r=R} \iint M_{\mu}^{0} d x^{3}
$$




\section{BELL-SZEKERES METRIC}

It is well-known that exact plane gravitational waves are simple time dependent plane symmetric solutions of the Einstein field equations [45]. The colliding plane wave spacetimes have been investigated extensively in GR [46] due to their interesting behaviour. The first exact solution of the Einstein-Maxwell equations representing colliding plane shock electromagnetic waves with co-linear polarizations was obtained by Bell and Szekeres [47]. This solution is conformally flat in the interaction region and is represented by the metric

$$
d s^{2}=2 d u d v+e^{-U}\left(e^{V} d x^{2}+e^{-V} d y^{2}\right),
$$

where the metric functions $U$ and $V$ depend on the null coordinates $u$ and $v$. The complete solution of the Einstein-Maxwell equations is

$$
\begin{aligned}
& U=-\log (f(u)+g(u)), \\
& V=\log (r w-p q)-\log (r w+p q),
\end{aligned}
$$

where

$$
\begin{aligned}
r & =\left(\frac{1}{2}+f\right)^{\frac{1}{2}}, \quad p=\left(\frac{1}{2}-f\right)^{\frac{1}{2}}, \\
w & =\left(\frac{1}{2}+g\right)^{\frac{1}{2}}, \quad q=\left(\frac{1}{2}-g\right)^{\frac{1}{2}}
\end{aligned}
$$

with

$$
f=\frac{1}{2}-\sin ^{2} P, \quad g=\frac{1}{2}-\sin ^{2} Q .
$$

Here $P=a u \theta(u), Q=b v \theta(v)$, where $\theta$ is the Heaviside unit step function, $a$ and $b$ are arbitrary constants.

The Cartesian form of the metric is found by substituting $t=u+v$ and $z=v-u$

$$
\begin{aligned}
d s^{2} & =\frac{1}{2} d t^{2}-\cos ^{2}\left\{\frac{1}{2} b(t-z) \theta\left(\frac{t-z}{2}\right)+\frac{1}{2} a(t+z) \theta\left(\frac{t+z}{2}\right)\right\} d x^{2} \\
& -\cos ^{2}\left\{\frac{1}{2} b(z-t) \theta\left(\frac{t-z}{2}\right)+\frac{1}{2} a(t+z) \theta\left(\frac{t+z}{2}\right)\right\} d y^{2}-\frac{1}{2} d z^{2}
\end{aligned}
$$

\section{A. Energy-Momentum Distribution in GR}

In this section, we find the energy-momentum distribution of Bell-Szekeres metric using Einstein, Landau-Lifshitz, Bergmann-Thomson and Möller's prescriptions in GR.

Using Einstein prescription, the components of the energymomentum density of Bell-Szekeres metric turn out to be

$$
\begin{aligned}
\Theta^{00} & =\frac{1}{32 \pi}\left[\cos \left\{b(t-z) \theta\left(\frac{t-z}{2}\right)\right\}\left\{2 b \theta\left(\frac{t-z}{2}\right)+b(t-z) \theta^{\prime}\left(\frac{t-z}{2}\right)\right\}^{2}\right. \\
& +b \sin \left\{b(t-z) \theta\left(\frac{t-z}{2}\right)\right\}\left\{4 \theta^{\prime}\left(\frac{t-z}{2}\right)+(t-z) \theta^{\prime \prime}\left(\frac{t-z}{2}\right)\right\} \\
& +a\left\{a \operatorname { c o s } \{ a ( t + z ) \theta ( \frac { t + z } { 2 } ) \} \left\{2 \theta\left(\frac{t+z}{2}\right)\right.\right. \\
& \left.+(t+z) \theta^{\prime}\left(\frac{t-z}{2}\right)\right\}^{2}+\sin \left\{a(t+z) \theta\left(\frac{t+z}{2}\right)\right\}\left\{4 \theta^{\prime}\left(\frac{t+z}{2}\right)\right. \\
& \left.\left.\left.+(t+z) \theta^{\prime \prime}\left(\frac{t+z}{2}\right)\right\}\right\}\right], \\
\Theta^{10} & =\Theta^{20}=0, \\
\Theta^{30} & =\frac{1}{32 \pi}\left[\cos \left\{b(t-z) \theta\left(\frac{t-z}{2}\right)\right\}\left\{2 b \theta\left(\frac{t-z}{2}\right)+b(t-z) \theta^{\prime}\left(\frac{t-z}{2}\right)\right\}^{2}\right. \\
& +b \sin \left\{b(t-z) \theta\left(\frac{t-z}{2}\right)\right\}\left\{4 \theta^{\prime}\left(\frac{t-z}{2}\right)+(t-z) \theta^{\prime \prime}\left(\frac{t-z}{2}\right)\right\} \\
& +a\left\{-a \cos \left\{a(t+z) \theta\left(\frac{t+z}{2}\right)\right\}\left\{2 \theta\left(\frac{t+z}{2}\right)\right.\right. \\
& +(t+z)\left\{\theta^{\prime}\left(\frac{t-z}{2}\right)\right\}^{2}-\sin \left\{a(t+z) \theta\left(\frac{t+z}{2}\right)\right\}\left\{4 \theta^{\prime}\left(\frac{t+z}{2}\right)\right. \\
& \left.\left.\left.\left.+(t+z) \theta^{\prime \prime}\left(\frac{t+z}{2}\right)\right\}\right\}\right\}\right] .
\end{aligned}
$$

When we use Landau-Lifshitz complex, we obtain the following energy-momentum density components 


$$
\begin{aligned}
& L^{00}=\frac{1}{256 \pi}\left[2 \cos \left\{2 b(t-z) \theta\left(\frac{t-z}{2}\right)\right\}\left\{2 b \theta\left(\frac{t-z}{2}\right)+b(t-z) \theta^{\prime}\left(\frac{t-z}{2}\right)\right\}^{2}\right. \\
& +b \sin \left\{2 b(t-z) \theta\left(\frac{t-z}{2}\right)\right\}\left\{4 \theta^{\prime}\left(\frac{t-z}{2}\right)+(t-z) \theta^{\prime \prime}\left(\frac{t-z}{2}\right)\right\} \\
& +2 b \sin \left\{b(t-z) \theta\left(\frac{t-z}{2}\right)\right\}\left\{2 a \operatorname { s i n } ( a ( t + z ) \theta ( \frac { t + z } { 2 } ) ) \left(2 \theta\left(\frac{t-z}{2}\right)\right.\right. \\
& \left.+(t-z) \theta^{\prime}\left(\frac{t-z}{2}\right)\right)\left(2 \theta\left(\frac{t-z}{2}\right)+(t+z) \theta^{\prime}\left(\frac{t-z}{2}\right)\right) \\
& \left.+\cos \left(a(t+z) \theta\left(\frac{t+z}{2}\right)\right)\left(4 \theta^{\prime}\left(\frac{t-z}{2}\right)+(t-z) \theta^{\prime \prime}\left(\frac{t-z}{2}\right)\right)\right\} \\
& +2 \cos \left\{b(t-z) \theta\left(\frac{t-z}{2}\right)\right\}\left\{\operatorname { c o s } \left\{a ( t + z ) \theta ( \frac { t + z } { 2 } ) \left\{4 a^{2} \theta^{2}\left(\frac{t+z}{2}\right)\right.\right.\right. \\
& +\left\{2 b \theta\left(\frac{t-z}{2}\right)+b(t-z) \theta^{\prime}\left(\frac{t-z}{2}\right)\right\}^{2}+4 a^{2}(t+z) \theta\left(\frac{t+z}{2}\right) \theta^{\prime}\left(\frac{t+z}{2}\right) \\
& \left.+a^{2}(t+z)^{2} \theta^{\prime}\left(\frac{t+z}{2}\right)^{2}\right\}+a \sin \left\{a(t+z) \theta\left(\frac{t+z}{2}\right)\right\}\left\{4 \theta^{\prime}\left(\frac{t+z}{2}\right)\right. \\
& \left.\left.\left.+(t+z) \theta^{\prime \prime}\left(\frac{t+z}{2}\right)\right\}\right\}\right\}+a\left\{2 a \operatorname { c o s } \{ 2 a ( t + z ) \theta ( \frac { t + z } { 2 } ) \} \left\{2 \theta\left(\frac{t+z}{2}\right)\right.\right. \\
& \left.+(t+z) \theta^{\prime}\left(\frac{t+z}{2}\right)\right\}^{2}+\sin \left\{2 a(t+z) \theta\left(\frac{t+z}{2}\right)\right\}\left\{4 \theta^{\prime}\left(\frac{t+z}{2}\right)\right. \\
& \left.\left.\left.+(t+z) \theta^{\prime \prime}\left(\frac{t+z}{2}\right)\right\}\right\}\right] \\
& L^{10}=L^{20}=0 \text {, } \\
& L^{30}=\frac{1}{256 \pi}\left[2 \cos \left\{2 b(t-z) \theta\left(\frac{t-z}{2}\right)\right\}\left\{2 b \theta\left(\frac{t-z}{2}\right)+b(t-z) \theta^{\prime}\left(\frac{t-z}{2}\right)\right\}^{2}\right. \\
& \left.+2 b \cos \left\{a(t+z) \theta\left(\frac{t+z}{2}\right)\right\} \sin \left\{b(t-z) \theta\left(\frac{t-z}{2}\right)\right)\right\}\left\{4 \theta^{\prime}\left(\frac{t-z}{2}\right)\right. \\
& \left.+(t-z) \theta^{\prime \prime}\left(\frac{t-z}{2}\right)\right\}+b \sin \left\{2 b(t-z) \theta\left(\frac{t-z}{2}\right)\right\}\left\{4 \theta^{\prime}\left(\frac{t-z}{2}\right)\right. \\
& \left.+(t-z) \theta^{\prime \prime}\left(\frac{t-z}{2}\right)\right\}+2 \cos \left\{b(t-z) \theta\left(\frac{t-z}{2}\right)\right\} \cos \{a(t \\
& \left.+z) \theta\left(\frac{t+z}{2}\right)\right\}\left\{2 b \theta\left(\frac{t-z}{2}\right)-2 a \theta\left(\frac{t+z}{2}\right)+b(t-z) \theta^{\prime}\left(\frac{t-z}{2}\right)\right. \\
& \left.-a(t+z) \theta^{\prime}\left(\frac{t+z}{2}\right)\right\}\left\{2 b \theta\left(\frac{t-z}{2}\right)+2 a \theta\left(\frac{t+z}{2}\right)+b(t-z) \theta^{\prime}\left(\frac{t-z}{2}\right)\right. \\
& \left.+a(t+z) \theta^{\prime}\left(\frac{t+z}{2}\right)\right\}-a \sin \left\{a(t+z) \theta\left(\frac{t+z}{2}\right)\right\}\left\{4 \theta^{\prime}\left(\frac{t+z}{2}\right)\right. \\
& \left.+(t+z) \theta^{\prime \prime}\left(\frac{t+z}{2}\right)\right\}+a\left\{-2 a \cos \left\{2 a(t+z) \theta\left(\frac{t+z}{2}\right)\right\}\left\{2 \theta\left(\frac{t+z}{2}\right)\right.\right. \\
& \left.+(t+z) \theta^{\prime}\left(\frac{t+z}{2}\right)\right\}^{2}-\sin \left\{2 a(t+z) \theta\left(\frac{t+z}{2}\right)\right\}\left\{4 \theta^{\prime}\left(\frac{t+z}{2}\right)\right. \\
& \left.\left.\left.+(t+z) \theta^{\prime \prime}\left(\frac{t+z}{2}\right)\right\}\right\}\right]
\end{aligned}
$$

The energy-momentum distribution in Bergmann-Thomson's prescription will become

$$
\begin{aligned}
B^{00} & =\frac{1}{32 \pi}\left[\cos \left\{b(t-z) \theta\left(\frac{t-z}{2}\right)\right\}\left\{2 b \theta\left(\frac{t-z}{2}\right)+b(t-z) \theta^{\prime}\left(\frac{t-z}{2}\right)\right\}^{2}\right. \\
& +b \sin \left\{b(t-z) \theta\left(\frac{t-z}{2}\right)\right\}\left\{4 \theta^{\prime}\left(\frac{t-z}{2}\right)+(t-z) \theta^{\prime \prime}\left(\frac{t-z}{2}\right)\right\} \\
& +a\left\{a \cos \left\{a(t+z) \theta\left(\frac{t+z}{2}\right)\right\}\left\{2 \theta\left(\frac{t+z}{2}\right)+(t+z) \theta^{\prime}\left(\frac{t-z}{2}\right)\right\}^{2}\right. \\
& \left.\left.+\sin \left\{a(t+z) \theta\left(\frac{t+z}{2}\right)\right\}\left\{4 \theta^{\prime}\left(\frac{t+z}{2}\right)+(t+z) \theta^{\prime \prime}\left(\frac{t+z}{2}\right)\right\}\right\}\right]
\end{aligned}
$$




$$
\begin{aligned}
B^{10} & =B^{20}=0 \\
B^{30} & =\frac{1}{32 \pi}\left[\cos \left\{b(t-z) \theta\left(\frac{t-z}{2}\right)\right\}\left\{2 b \theta\left(\frac{t-z}{2}\right)+b(t-z) \theta^{\prime}\left(\frac{t-z}{2}\right)\right\}^{2}\right. \\
& +b \sin \left\{b(t-z) \theta\left(\frac{t-z}{2}\right)\right\}\left\{4 \theta^{\prime}\left(\frac{t-z}{2}\right)+(t-z) \theta^{\prime \prime}\left(\frac{t-z}{2}\right)\right\} \\
& +a\left\{-a \cos \left\{a(t+z) \theta\left(\frac{t+z}{2}\right)\right\}\left\{2 \theta\left(\frac{t+z}{2}\right)+(t+z)\left\{\theta^{\prime}\left(\frac{t-z}{2}\right)\right\}^{2}\right.\right. \\
& -\sin \left\{a(t+z) \theta\left(\frac{t+z}{2}\right)\right\}\left\{4 \theta^{\prime}\left(\frac{t+z}{2}\right)\right. \\
& \left.\left.\left.\left.+(t+z) \theta^{\prime \prime}\left(\frac{t+z}{2}\right)\right\}\right\}\right\}\right] .
\end{aligned}
$$

Finally, energy and momentum densities in Möller's prescription take the form

$$
M^{a 0}=0, \quad(a=0,1,2,3)
$$

\section{B. Energy-Momentum Distribution in TPT}

The tetrad components, in Cartesian coordinates, of Eq.(38) are given as

$$
\begin{aligned}
& h_{0}^{0}=\frac{1}{\sqrt{2}} \\
& h_{1}^{1}=\cos \left\{\frac{1}{2} b(t-z) \theta\left(\frac{t-z}{2}\right)+\frac{1}{2} a(t+z) \theta\left(\frac{t+z}{2}\right)\right\} \\
& h_{2}^{2}=\cos \left\{\frac{1}{2} b(t-z) \theta\left(\frac{t-z}{2}\right)-\frac{1}{2} a(t+z) \theta\left(\frac{t+z}{2}\right)\right\} \\
& h_{3}^{3}=\frac{1}{\sqrt{2}}
\end{aligned}
$$

and its inverse is

$$
\begin{aligned}
& h_{0}{ }^{0}=\sqrt{2} \\
& h_{1}{ }^{1}=\sec \left[\frac{1}{2}\left\{b(t-z) \theta\left(\frac{t-z}{2}\right)+a(t+z) \theta\left(\frac{t+z}{2}\right)\right\}\right], \\
& h_{2}{ }^{2}=\sec \left[\frac{1}{2}\left\{b(t-z) \theta\left(\frac{t-z}{2}\right)-a(t+z) \theta\left(\frac{t+z}{2}\right)\right\}\right], \\
& h_{3}{ }^{3}=\sqrt{2} .
\end{aligned}
$$

Using these values, we can find the Weitzenböck connections and the corresponding torsion tensor. These are then used to find the components of the superpotential that are essential to obtain energy-momentum density components. When we make use of the components of superpotential in Eqs.(16)(18), the energy-momentum density components of Einstein, Landau-Lifshitz and Bergmann-Thomson become

$$
\begin{aligned}
h E^{00} & =\frac{1}{32 \pi}\left[\cos \left\{b(t-z) \theta\left(\frac{t-z}{2}\right)\right\}\left\{2 b \theta\left(\frac{t-z}{2}\right)+b(t-z) \theta^{\prime}\left(\frac{t-z}{2}\right)\right\}^{2}\right. \\
& +b \sin \left\{b(t-z) \theta\left(\frac{t-z}{2}\right)\right\}\left\{4 \theta^{\prime}\left(\frac{t-z}{2}\right)+(t-z) \theta^{\prime \prime}\left(\frac{t-z}{2}\right)\right\} \\
& +a\left\{a \operatorname { c o s } \{ a ( t + z ) \theta ( \frac { t + z } { 2 } ) \} \left\{2 \theta\left(\frac{t+z}{2}\right)\right.\right. \\
& \left.+(t+z) \theta^{\prime}\left(\frac{t-z}{2}\right)\right\}^{2}+\sin \left\{a(t+z) \theta\left(\frac{t+z}{2}\right)\right\}\left\{4 \theta^{\prime}\left(\frac{t+z}{2}\right)\right. \\
& \left.\left.\left.+(t+z) \theta^{\prime \prime}\left(\frac{t+z}{2}\right)\right\}\right\}\right], \\
h E^{10} & =h E^{20}=0,
\end{aligned}
$$




$$
\begin{aligned}
h E^{30} & =\frac{1}{32 \pi}\left[\cos \left\{b(t-z) \theta\left(\frac{t-z}{2}\right)\right\}\left\{2 b \theta\left(\frac{t-z}{2}\right)+b(t-z) \theta^{\prime}\left(\frac{t-z}{2}\right)\right\}^{2}\right. \\
& +b \sin \left\{b(t-z) \theta\left(\frac{t-z}{2}\right)\right\}\left\{4 \theta^{\prime}\left(\frac{t-z}{2}\right)+(t-z) \theta^{\prime \prime}\left(\frac{t-z}{2}\right)\right\} \\
& +a\left\{-a \cos \left\{a(t+z) \theta\left(\frac{t+z}{2}\right)\right\}\left\{2 \theta\left(\frac{t+z}{2}\right)\right.\right. \\
& +(t+z)\left\{\theta^{\prime}\left(\frac{t-z}{2}\right)\right\}^{2}-\sin \left\{a(t+z) \theta\left(\frac{t+z}{2}\right)\right\}\left\{4 \theta^{\prime}\left(\frac{t+z}{2}\right)\right. \\
& \left.\left.\left.\left.+(t+z) \theta^{\prime \prime}\left(\frac{t+z}{2}\right)\right\}\right\}\right\}\right],
\end{aligned}
$$

$$
\begin{aligned}
& h L^{00}=\frac{1}{256 \pi}\left[2 \cos \left\{2 b(t-z) \theta\left(\frac{t-z}{2}\right)\right\}\left\{2 b \theta\left(\frac{t-z}{2}\right)+b(t-z) \theta^{\prime}\left(\frac{t-z}{2}\right)\right\}^{2}\right. \\
& +b \sin \left\{2 b(t-z) \theta\left(\frac{t-z}{2}\right)\right\}\left\{4 \theta^{\prime}\left(\frac{t-z}{2}\right)+(t-z) \theta^{\prime \prime}\left(\frac{t-z}{2}\right)\right\} \\
& +2 b \sin \left\{b(t-z) \theta\left(\frac{t-z}{2}\right)\right\}\left\{2 a \operatorname { s i n } \{ a ( t + z ) \theta ( \frac { t + z } { 2 } ) \} \left\{2 \theta\left(\frac{t-z}{2}\right)\right.\right. \\
& \left.+(t-z) \theta^{\prime}\left(\frac{t-z}{2}\right)\right\}\left\{2 \theta\left(\frac{t-z}{2}\right)+(t+z) \theta^{\prime}\left(\frac{t-z}{2}\right)\right\} \\
& \left.+\cos \left\{a(t+z) \theta\left(\frac{t+z}{2}\right)\right\}\left\{4 \theta^{\prime}\left(\frac{t-z}{2}\right)+(t-z) \theta^{\prime \prime}\left(\frac{t-z}{2}\right)\right\}\right\} \\
& +2 \cos \left\{b(t-z) \theta\left(\frac{t-z}{2}\right)\right\}\left\{\operatorname { c o s } \left\{a ( t + z ) \theta ( \frac { t + z } { 2 } ) \left\{4 a^{2} \theta^{2}\left(\frac{t+z}{2}\right)\right.\right.\right. \\
& +\left\{2 b \theta\left(\frac{t-z}{2}\right)+b(t-z) \theta^{\prime}\left(\frac{t-z}{2}\right)\right\}^{2}+4 a^{2}(t+z) \theta\left(\frac{t+z}{2}\right) \theta^{\prime}\left(\frac{t+z}{2}\right) \\
& \left.+a^{2}(t+z)^{2} \theta^{\prime}\left(\frac{t+z}{2}\right)^{2}\right\}+a \sin \left\{a ( t + z ) \theta ( \frac { t + z } { 2 } ) \left\{4 \theta^{\prime}\left(\frac{t+z}{2}\right)\right.\right. \\
& \left.\left.+(t+z) \theta^{\prime \prime}\left(\frac{t+z}{2}\right)\right\}\right\}+a\left\{2 a \operatorname { c o s } \{ 2 a ( t + z ) \theta ( \frac { t + z } { 2 } ) \} \left\{2 \theta\left(\frac{t+z}{2}\right)\right.\right. \\
& \left.+(t+z) \theta^{\prime}\left(\frac{t+z}{2}\right)\right\}^{2}+\sin \left\{2 a ( t + z ) \theta ( \frac { t + z } { 2 } ) \left\{4 \theta^{\prime}\left(\frac{t+z}{2}\right)\right.\right. \\
& \left.\left.\left.\left.\left.\left.+(t+z) \theta^{\prime \prime}\left(\frac{t+z}{2}\right)\right\}\right\}\right\}\right\}\right\}\right], \\
& h L^{10}=h L^{20}=0 \text {, } \\
& h L^{30}=\frac{1}{256 \pi}\left[2 \cos \left\{2 b(t-z) \theta\left(\frac{t-z}{2}\right)\right\}\left\{2 b \theta\left(\frac{t-z}{2}\right)+b(t-z) \theta^{\prime}\left(\frac{t-z}{2}\right)\right\}^{2}\right. \\
& +2 b \cos \left\{a(t+z) \theta\left(\frac{t+z}{2}\right)\right\} \sin \left\{b(t-z) \theta\left(\frac{t-z}{2}\right)\right\}\left\{4 \theta^{\prime}\left(\frac{t-z}{2}\right)\right. \\
& \left.+(t-z) \theta^{\prime \prime}\left(\frac{t-z}{2}\right)\right\}+b \sin \left\{2 b(t-z) \theta\left(\frac{t-z}{2}\right)\right\}\left\{4 \theta^{\prime}\left(\frac{t-z}{2}\right)+(t-z) \theta^{\prime \prime}\left(\frac{t-z}{2}\right)\right\} \\
& +2 \cos \left\{b(t-z) \theta\left(\frac{t-z}{2}\right)\right\} \cos \left\{a(t+z) \theta\left(\frac{t+z}{2}\right)\right\}\left\{2 b \theta\left(\frac{t-z}{2}\right)-2 a \theta\left(\frac{t+z}{2}\right)\right. \\
& \left.+b(t-z) \theta^{\prime}\left(\frac{t-z}{2}\right)-a(t+z) \theta^{\prime}\left(\frac{t+z}{2}\right)\right\}\left(2 b \theta\left(\frac{t-z}{2}\right)+2 a \theta\left(\frac{t+z}{2}\right)\right. \\
& \left.+b(t-z) \theta^{\prime}\left(\frac{t-z}{2}\right)+a(t+z) \theta^{\prime}\left(\frac{t+z}{2}\right)\right)-a \sin \left\{a(t+z) \theta\left(\frac{t+z}{2}\right)\right\}\left\{4 \theta^{\prime}\left(\frac{t+z}{2}\right)\right. \\
& \left.+(t+z) \theta^{\prime \prime}\left(\frac{t+z}{2}\right)\right\}+a\left\{-2 a \cos \left\{2 a(t+z) \theta\left(\frac{t+z}{2}\right)\right\}\left\{2 \theta\left(\frac{t+z}{2}\right)\right.\right. \\
& \left.+(t+z) \theta^{\prime}\left(\frac{t+z}{2}\right)\right\}^{2}-\sin \left\{2 a(t+z) \theta\left(\frac{t+z}{2}\right)\right\}\left\{4 \theta^{\prime}\left(\frac{t+z}{2}\right)\right. \\
& \left.\left.\left.+(t+z) \theta^{\prime \prime}\left(\frac{t+z}{2}\right)\right\}\right\}\right],
\end{aligned}
$$

and

$$
\begin{aligned}
h B^{00} & =\frac{1}{32 \pi}\left[\cos \left\{b(t-z) \theta\left(\frac{t-z}{2}\right)\right\}\left\{2 b \theta\left(\frac{t-z}{2}\right)+b(t-z) \theta^{\prime}\left(\frac{t-z}{2}\right)\right\}^{2}\right. \\
& +b \sin \left\{b(t-z) \theta\left(\frac{t-z}{2}\right)\right\}\left\{4 \theta^{\prime}\left(\frac{t-z}{2}\right)+(t-z) \theta^{\prime \prime}\left(\frac{t-z}{2}\right)\right\}
\end{aligned}
$$




$$
\begin{aligned}
+ & a\left\{a \operatorname { c o s } ( a ( t + z ) \theta ( \frac { t + z } { 2 } ) ) \left(2 \theta\left(\frac{t+z}{2}\right)\right.\right. \\
+ & \left.(t+z) \theta^{\prime}\left(\frac{t-z}{2}\right)\right)^{2}+\sin \left(a(t+z) \theta\left(\frac{t+z}{2}\right)\right)\left\{4 \theta^{\prime}\left(\frac{t+z}{2}\right)\right. \\
& \left.\left.\left.+(t+z) \theta^{\prime \prime}\left(\frac{t+z}{2}\right)\right\}\right\}\right], \\
h B^{10} & =h B^{20}=0, \\
h B^{30} & =\frac{1}{32 \pi}\left[\cos \left\{b(t-z) \theta\left(\frac{t-z}{2}\right)\right\}\left\{2 b \theta\left(\frac{t-z}{2}\right)+b(t-z) \theta^{\prime}\left(\frac{t-z}{2}\right)\right\}^{2}\right. \\
& +b \sin \left\{b(t-z) \theta\left(\frac{t-z}{2}\right)\right\}\left\{4 \theta^{\prime}\left(\frac{t-z}{2}\right)+(t-z) \theta^{\prime \prime}\left(\frac{t-z}{2}\right)\right\} \\
& +a\left\{-a \cos \left\{a(t+z) \theta\left(\frac{t+z}{2}\right)\right\}\left\{2 \theta\left(\frac{t+z}{2}\right)\right.\right. \\
& +(t+z)\left(\theta^{\prime}\left(\frac{t-z}{2}\right)\right)^{2}-\sin \left\{a(t+z) \theta\left(\frac{t+z}{2}\right)\right\}\left\{4 \theta^{\prime}\left(\frac{t+z}{2}\right)\right. \\
& \left.\left.\left.\left.+(t+z) \theta^{\prime \prime}\left(\frac{t+z}{2}\right)\right\}\right\}\right\}\right],
\end{aligned}
$$

respectively.

Similarly, we can proceed to find energy-momentum density components using Möller's prescription in TPT. The tetrad components of Eq.(34) are given as

$$
h^{a}{ }_{\mu}=\left(\begin{array}{cccc}
1 & \frac{1}{2} & 0 & 0 \\
-1 & \frac{1}{2} & 0 & 0 \\
0 & 0 & \cos \{a u \theta(u)+b \nu \theta(v)\} & 0 \\
0 & 0 & 0 & \cos \{a u \theta(u)-b v \theta(v)\}
\end{array}\right)
$$

and its inverse

$$
h_{a}{ }^{\mu}=\left(\begin{array}{cccc}
\frac{1}{2} & 1 & 0 & 0 \\
-\frac{1}{2} & 1 & 0 & 0 \\
0 & 0 & \sec \{a u \theta(u)+b v \theta(v)\} & 0 \\
0 & 0 & 0 & \sec \{a u \theta(u)-b v \theta(v)\}
\end{array}\right)
$$

Consequently, the energy-momentum density components turn out to be

$$
\begin{aligned}
M^{00} & =M^{20}=M^{30}=0, \\
M^{10} & =\frac{a}{\kappa}\left[2 a \cos \{2 a u \theta(u)\}\left\{\theta(u)+u \theta^{\prime}(u)\right\}^{2}\right. \\
& \left.+\sin \{2 a u \theta(u)\}\left\{2 \theta^{\prime}(u)+u \theta^{\prime \prime}(u)\right\}\right] .
\end{aligned}
$$

\section{DISCUSSION}

The problem of energy-momentum localization has been a subject of many researchers but still remains un-resolved. Numerous attempts have been made to explore a quantity which describes the distribution of energy-momentum due to matter, non-gravitational and gravitational fields. This paper continues the investigation of comparing various distributions presented in the literature in the framework of GR and TPT. The more information that is assembled on the subject, the better. We have used four different prescriptions namely Einstein, Landau-Lifshitz, Bergmann and Möller to calculate energy- momentum distribution of a Bell-Szekeres metric in the context of both GR and TPT. The resulting non-vanishing components of the energy-momentum density are displayed in the following tables. In these tables, EMD will stand for energymomentum density.

Energy and momentum become constant for Möller's prescription in GR.

\begin{tabular}{|l|l|}
\hline EMD & Expression \\
\hline$M^{10}$ & $\frac{a}{\kappa}\left[2 a \cos \{2 a u \theta(u)\}\left\{\theta(u)+u \theta^{\prime}(u)\right\}^{2}\right.$ \\
& $\left.+\sin \{2 a u \theta(u)\}\left\{2 \theta^{\prime}(u)+u \theta^{\prime \prime}(u)\right\}\right]$ \\
\hline
\end{tabular}

Notice that energy is constant in both GR and TPT. 
From these tables, it follows that the energy-momentum density components turn out to be finite and well-defined in each case. It is interesting to note that we obtain same results for all the four prescriptions used here both in GR and TPT. We find constant energy for Möller's prescription in both these theories. The summary of the results is the following:

$$
\begin{aligned}
E^{a 0} & =h E^{a 0}=B^{a 0}=h B^{a 0}, \\
L^{a 0} & =h L^{a 0}, \\
M_{0}^{0} & =h M_{0}^{0}, \quad(a=0,1,2,3) .
\end{aligned}
$$

This indicates that both GR and TPT are equivalent theories for Einstein, Bergmann-Thomson, Landau-Lifshitz and Möller's prescriptions.

It has been shown [48] that for a given spacetime many quasi-local mass definitions do not give agreed results. In GR, several energy-momentum expressions (reference frame dependent pseudo-tensors) have been proposed. All this makes it difficult to decide which one to use, and raises a suspicion that they could give different energy-momentum distributions for one fixed spacetime. We can conclude that the use of the energy-momentum complexes may not be sufficient to find the energy-momentum distribution of the physical systems.

Finally, we would like to mention here that the tetrad formalism itself has some advantages. These advantages come mainly from the independence of the tetrad formalism from the equivalence principle and consequent suitability to the discussion of quantum issues. Some classic solutions of the field equations have already been translated into the teleparallel language. Thus TPT seems to provide a more appropriate environment to deal with the energy problem.
[1] A. Trautman, Gravitation: An Introduction to Current Research ed. Witten, L. (Wiley, New York, 1962)169.

[2] L.D. Landau and E.M. Lifshitz, The Classical Theory of Fields (Addison-Wesley Press, New York, 1962).

[3] A. Papapetrou, Proc. R. Irish Acad. A 52, 11 (1948).

[4] P.G. Bergmann and R. Thompson, Phys. Rev. D 89, 400 (1958).

[5] C. Möller, Ann. Phys. (NY) 4, 347 (1958).

[6] S. Chandrasekhar and V. Ferrari, Proc. Roy. Soc. London A 435, 645 (1991).

[7] G. Bergqvist, Class. Quantum Gravit. 9, 1753 (1992).

[8] G. Bergqvist, Class. Quantum Gravit. 9, 1917 (1992).

[9] J.M. Nester and C. Chen, Class. Quantum Gravit. 16, 1279 (1999).

[10] R. Penrose, Proc. Roy. Soc. London A388(1982)457; GR 10 Conference eds. B. Bertotti, F. de Felice, and A. I. Pascolini, (Padova, 1983) 607.

[11] J.D. Brown and J.W. York, Jr. Phys. Rev. D 47, 1407 (1993).

[12] C.C. Chang, J.M. Nester, and C. Chen, Phys. Rev. Lett. 83, 1897 (1999).

[13] K.S. Virbhadra, Phys. Rev. D 41, 1086(1990); D 42, 1066 (1990) and references therein.

[14] K.S. Virbhadra, Phys. Rev. D 60, 104041 (1999).

[15] K.S. Virbhadra, Phys. Rev. D 42, 2919 (1990).

[16] N. Rosen and K.S. Virbhadra, Gen. Relativ. Gravit. 25, 429 (1993).

[17] J.M. Aguirregabiria, A. Chamorro, and K.S. Virbhadra, Gen. Relativ. Gravit. 28, 1393 (1996).

[18] S.S. Xulu, Int. J. Mod. Phys. A 15, 2979 (2000); Mod. Phys. Lett. A 15, 1151 (2000) and reference therein.

[19] S.S. Xulu, Astrophys. Space Sci. 283, 23 (2003).

[20] A. Chamorro and K.S. Virbhardra, Int. J. Mod. Phys. D 5, 251 (1994).

[21] S.S. Xulu, Int. J. Mod. Phys. D 7, 773 (1998).

[22] I. Ramdinschi and I.C. Yang, On the Energy of String Black Holes, New Developments in String Theory Research ed.
Grece, A. (New York, Nova Science, 2005).

[23] E.C. Vagenas, Int. J. Mod. Phys. A 18, 5781 (2003).

[24] R.M. Gad, Astrophys. Space Sci. 295, 459 (2005).

[25] S.S. Xulu, Int. J. Theor. Phys. 37, 1773 (2003).

[26] F.I. Cooperstock, Mod. Phys. Lett. A 14, 1531 (1999).

[27] N. Rosen, Gen. Relativ. Gravit. 26, 323 (1994).

[28] N. Banerjee and S. Sen, Pramana J. Phys. 49, 609 (1997).

[29] M. Sharif, Int. J. Mod. Phys. A 17, 1175 (2002).

[30] M. Sharif, Int. J. Mod. Phys. A 18, 4361 (2003).

[31] M. Sharif and T. Fatima, Int. J. Mod. Phys. A 20, 4309 (2005).

[32] M. Sharif, and T. Fatima, Nouvo Cim. B 120, 533 (2005).

[33] F.I. Mikhail, M.I. Wanas, A. Hindawi, and E.I. Lashin, Int. J. Theo. Phys. 32, 1627 (1993).

[34] G.G.L. Nashed, Nouvo Cim. B 117, 521 (2002).

[35] C. Möller, Tetrad Fields and Conservation Laws in General Relativity (Academic Press, London, 1962).

[36] T. Vargas, Gen. Relativ. Gravit. 30, 1255 (2004).

[37] L. Loi So and T. Vargas, Chinese J. Phys. 43, 901 (2005).

[38] M. Salti and A. Havare, Int. J. Mod. Phys. A 20, 2169 (2005).

[39] O. Aydogdu and M. Salti, Astrophys. Space Sci. 229, 227 (2005).

[40] M. Sharif and M.J. Amir, Mod. Phys. Lett. A 22, 425 (2007).

[41] M. Sharif and M. Azam, Int. J. Mod. Phys. A 22, 1935 (2007).

[42] C. Möller, Ann. Phys. (NY) 12, 118 (1961).

[43] R. Aldrovendi and J.G. Pereira, An Introduction to Gravitation Theory (preprint)

[44] R. Aldrovandi and J.C. Pereira, An Introduction to Geometrical Physics (World Scientific, 1995).

[45] O.R. Baldwin and G.B. Jeffrey, Proc. R. Soc. London A 111, 95 (1926).

[46] J.B. Griffiths, Colliding Plane Waves in General Relativity (Clarendon Press, Oxford, 1991).

[47] P. Bell and P. Szekeres, Gen. Relativ. Gravit. 5, 275 (1974).

[48] G. Bergqvist, Class. Quantum Gravit. 9, 1753 (1992). 
Table 1(a) Bell-Szekeres Metric: Einstein's Prescription in GR and TPT \begin{tabular}{|l|l}
\hline EMD & Expression
\end{tabular}

\begin{tabular}{|c|c|}
\hline \multirow{3}{*}{$\Theta^{00}$} & $\frac{1}{32 \pi}\left[\cos \left\{b(t-z) \theta\left(\frac{t-z}{2}\right)\right\}\left\{2 b \theta\left(\frac{t-z}{2}\right)+b(t-z) \theta^{\prime}\left(\frac{t-z}{2}\right)\right\}^{2}\right.$ \\
& $+b \sin \left\{b(t-z) \theta\left(\frac{t-z}{2}\right)\right\}\left\{4 \theta^{\prime}\left(\frac{t-z}{2}\right)+(t-z) \theta^{\prime \prime}\left(\frac{t-z}{2}\right)\right\}$ \\
& $+a\left\{a \cos \left\{a(t+z) \theta\left(\frac{t+z}{2}\right)\right\}\left\{2 \theta\left(\frac{t+z}{2}\right)+(t+z) \theta^{\prime}\left(\frac{t-z}{2}\right)\right\}^{2}\right.$ \\
& $\left.\left.+\sin \left\{a(t+z) \theta\left(\frac{t+z}{2}\right)\right\}\left\{4 \theta^{\prime}\left(\frac{t+z}{2}\right)+(t+z) \theta^{\prime \prime}\left(\frac{t+z}{2}\right)\right\}\right\}\right]$ \\
\hline \multirow{3}{*}{$\Theta^{30}$} & $\frac{1}{32 \pi}\left[\cos \left\{b(t-z) \theta\left(\frac{t-z}{2}\right)\right\}\left\{2 b \theta\left(\frac{t-z}{2}\right)+b(t-z) \theta^{\prime}\left(\frac{t-z}{2}\right)\right\}^{2}\right.$ \\
& $+b \sin \left\{b(t-z) \theta\left(\frac{t-z}{2}\right)\right\}\left\{4 \theta^{\prime}\left(\frac{t-z}{2}\right)+(t-z) \theta^{\prime \prime}\left(\frac{t-z}{2}\right)\right\}$ \\
& $+a\left\{-a \cos \left\{a(t+z) \theta\left(\frac{t+z}{2}\right)\right\}\left\{2 \theta\left(\frac{t+z}{2}\right)+(t+z)\left\{\theta^{\prime}\left(\frac{t-z}{2}\right)\right\}^{2}\right.\right.$ \\
& $\left.\left.\left.-\sin \left\{a(t+z) \theta\left(\frac{t+z}{2}\right)\right\}\left\{4 \theta^{\prime}\left(\frac{t+z}{2}\right)+(t+z) \theta^{\prime \prime}\left(\frac{t+z}{2}\right)\right\}\right\}\right\}\right]$ \\
\hline
\end{tabular}

\section{EMD Expression}

\begin{tabular}{|c|c|}
\hline \multirow{4}{*}{$h E^{00}$} & $\frac{1}{32 \pi}\left[\cos \left\{b(t-z) \theta\left(\frac{t-z}{2}\right)\right\}\left\{2 b \theta\left(\frac{t-z}{2}\right)+b(t-z) \theta^{\prime}\left(\frac{t-z}{2}\right)\right\}^{2}\right.$ \\
& $+b \sin \left\{b(t-z) \theta\left(\frac{t-z}{2}\right)\right\}\left\{4 \theta^{\prime}\left(\frac{t-z}{2}\right)+(t-z) \theta^{\prime \prime}\left(\frac{t-z}{2}\right)\right\}$ \\
& $+a\left\{a \cos \left\{a(t+z) \theta\left(\frac{t+z}{2}\right)\right\}\left\{2 \theta\left(\frac{t+z}{2}\right)+(t+z) \theta^{\prime}\left(\frac{t-z}{2}\right)\right\}^{2}\right.$ \\
& $\left.\left.+\sin \left\{a(t+z) \theta\left(\frac{t+z}{2}\right)\right\}\left\{4 \theta^{\prime}\left(\frac{t+z}{2}\right)+(t+z) \theta^{\prime \prime}\left(\frac{t+z}{2}\right)\right\}\right\}\right]$ \\
\hline \multirow{4}{*}{$h E^{30}$} & $\frac{1}{32 \pi}\left[\cos \left\{b(t-z) \theta\left(\frac{t-z}{2}\right)\right\}\left\{2 b \theta\left(\frac{t-z}{2}\right)+b(t-z) \theta^{\prime}\left(\frac{t-z}{2}\right)\right\}^{2}\right.$ \\
& $+b \sin \left\{b(t-z) \theta\left(\frac{t-z}{2}\right)\right\}\left\{4 \theta^{\prime}\left(\frac{t-z}{2}\right)+(t-z) \theta^{\prime \prime}\left(\frac{t-z}{2}\right)\right\}$ \\
& $+a\left\{-a \cos \left\{a(t+z) \theta\left(\frac{t+z}{2}\right)\right\}\left\{2 \theta\left(\frac{t+z}{2}\right)+(t+z)\left\{\theta^{\prime}\left(\frac{t-z}{2}\right)\right\}^{2}\right.\right.$ \\
& $\left.\left.\left.-\sin \left\{a(t+z) \theta\left(\frac{t+z}{2}\right)\right\}\left\{4 \theta^{\prime}\left(\frac{t+z}{2}\right)+(t+z) \theta^{\prime \prime}\left(\frac{t+z}{2}\right)\right\}\right\}\right\}\right]$ \\
\hline
\end{tabular}

Table 1(b) Bell-Szekeres Metric: Landau-Lifshitz's Prescription in GR and TPT

\begin{tabular}{|c|c|}
\hline EMD & \\
\hline$L^{00}$ & $\begin{array}{c}\frac{1}{256 \pi}\left[2 \cos \left\{2 b(t-z) \theta\left(\frac{t-z}{2}\right)\right\}\left\{2 b \theta\left(\frac{t-z}{2}\right)+b(t-z) \theta^{\prime}\left(\frac{t-z}{2}\right)\right\}^{2}\right. \\
+b \sin \left\{2 b(t-z) \theta\left(\frac{t-z}{2}\right)\right\}\left\{4 \theta^{\prime}\left(\frac{t-z}{2}\right)+(t-z) \theta^{\prime \prime}\left(\frac{t-z}{2}\right)\right\} \\
+2 b \sin \left\{b(t-z) \theta\left(\frac{t-z}{2}\right)\right\}\left\{2 a \sin \left(a(t+z) \theta\left(\frac{t+z}{2}\right)\right)\left(2 \theta\left(\frac{t-z}{2}\right)\right.\right. \\
\left.+(t-z) \theta^{\prime}\left(\frac{t-z}{2}\right)\right)\left(2 \theta\left(\frac{t-z}{2}\right)+(t+z) \theta^{\prime}\left(\frac{t-z}{2}\right)\right)+\cos (a(t \\
\left.\left.+z) \theta\left(\frac{t+z}{2}\right)\right)\left(4 \theta^{\prime}\left(\frac{t-z}{2}\right)+(t-z) \theta^{\prime \prime}\left(\frac{t-z}{2}\right)\right)\right\}+2 \cos \{b(t \\
\left.-z) \theta\left(\frac{t-z}{2}\right)\right\}\left\{\cos \left\{a(t+z) \theta\left(\frac{t+z}{2}\right)\left\{4 a^{2} \theta^{2}\left(\frac{t+z}{2}\right)+\left\{2 b \theta\left(\frac{t-z}{2}\right)\right.\right.\right.\right. \\
\left.+b(t-z) \theta^{\prime}\left(\frac{t-z}{2}\right)\right\}^{2}+4 a^{2}(t+z) \theta\left(\frac{t+z}{2}\right) \theta^{\prime}\left(\frac{t+z}{2}\right)+a^{2}(t+z)^{2} \\
\left.\theta^{\prime}\left(\frac{t+z}{2}\right)^{2}\right\}+a \sin \left\{a(t+z) \theta\left(\frac{t+z}{2}\right)\right\}\left\{4 \theta^{\prime}\left(\frac{t+z}{2}\right)\right. \\
\left.\left.\left.+(t+z) \theta^{\prime \prime}\left(\frac{t+z}{2}\right)\right\}\right\}\right\} \\
+a\left\{2 a \cos \left\{2 a(t+z) \theta\left(\frac{t+z}{2}\right)\right\}\left\{2 \theta\left(\frac{t+z}{2}\right)+(t+z) \theta^{\prime}\left(\frac{t+z}{2}\right)\right\}^{2}\right. \\
\left.\left.+\sin \left\{2 a(t+z) \theta\left(\frac{t+z}{2}\right)\right\}\left\{4 \theta^{\prime}\left(\frac{t+z}{2}\right)+(t+z) \theta^{\prime \prime}\left(\frac{t+z}{2}\right)\right\}\right\}\right]\end{array}$ \\
\hline$L^{30}$ & $\begin{array}{c}\frac{1}{256 \pi}\left[2 \cos \left\{2 b(t-z) \theta\left(\frac{t-z}{2}\right)\right\}\left\{2 b \theta\left(\frac{t-z}{2}\right)+b(t-z) \theta^{\prime}\left(\frac{t-z}{2}\right)\right\}^{2}\right. \\
\left.+2 b \cos \left\{a(t+z) \theta\left(\frac{t+z}{2}\right)\right\} \sin \left\{b(t-z) \theta\left(\frac{t-z}{2}\right)\right)\right\}\left\{4 \theta^{\prime}\left(\frac{t-z}{2}\right)\right. \\
\left.+(t-z) \theta^{\prime \prime}\left(\frac{t-z}{2}\right)\right\} \\
+b \sin \left\{2 b(t-z) \theta\left(\frac{t-z}{2}\right)\right\}\left\{4 \theta^{\prime}\left(\frac{t-z}{2}\right)+(t-z) \theta^{\prime \prime}\left(\frac{t-z}{2}\right)\right\} \\
\quad+2 \cos \left\{b(t-z) \theta\left(\frac{t-z}{2}\right)\right\} \cos \left\{a(t+z) \theta\left(\frac{t+z}{2}\right)\right\} \\
\left\{2 b \theta^{\prime}\left(\frac{t-z}{2}\right)-2 a \theta\left(\frac{t+z}{2}\right)+b(t-z) \theta^{\prime}\left(\frac{t-z}{2}\right)-a(t+z) \theta^{\prime}\left(\frac{t+z}{2}\right)\right\} \\
\left\{2 b \theta\left(\frac{t-z}{2}\right)+2 a \theta\left(\frac{t+z}{2}\right)+b(t-z) \theta^{\prime}\left(\frac{t-z}{2}\right)+a(t+z) \theta^{\prime}\left(\frac{t+z}{2}\right)\right\} \\
\quad-a \sin \left\{a(t+z) \theta\left(\frac{t+z}{2}\right)\right\}\left\{4 \theta^{\prime}\left(\frac{t+z}{2}\right)+(t+z) \theta^{\prime \prime}\left(\frac{t+z}{2}\right)\right\} \\
+a\left\{-2 a \cos \left\{2 a(t+z) \theta\left(\frac{t+z}{2}\right)\right\}\left\{2 \theta\left(\frac{t+z}{2}\right)+(t+z) \theta^{\prime}\left(\frac{t+z}{2}\right)\right\}^{2}\right. \\
\left.\left.\quad-\sin \left\{2 a(t+z) \theta\left(\frac{t+z}{2}\right)\right\}\left\{4 \theta^{\prime}\left(\frac{t+z}{2}\right)+(t+z) \theta^{\prime \prime}\left(\frac{t+z}{2}\right)\right\}\right\}\right]\end{array}$ \\
\hline
\end{tabular}




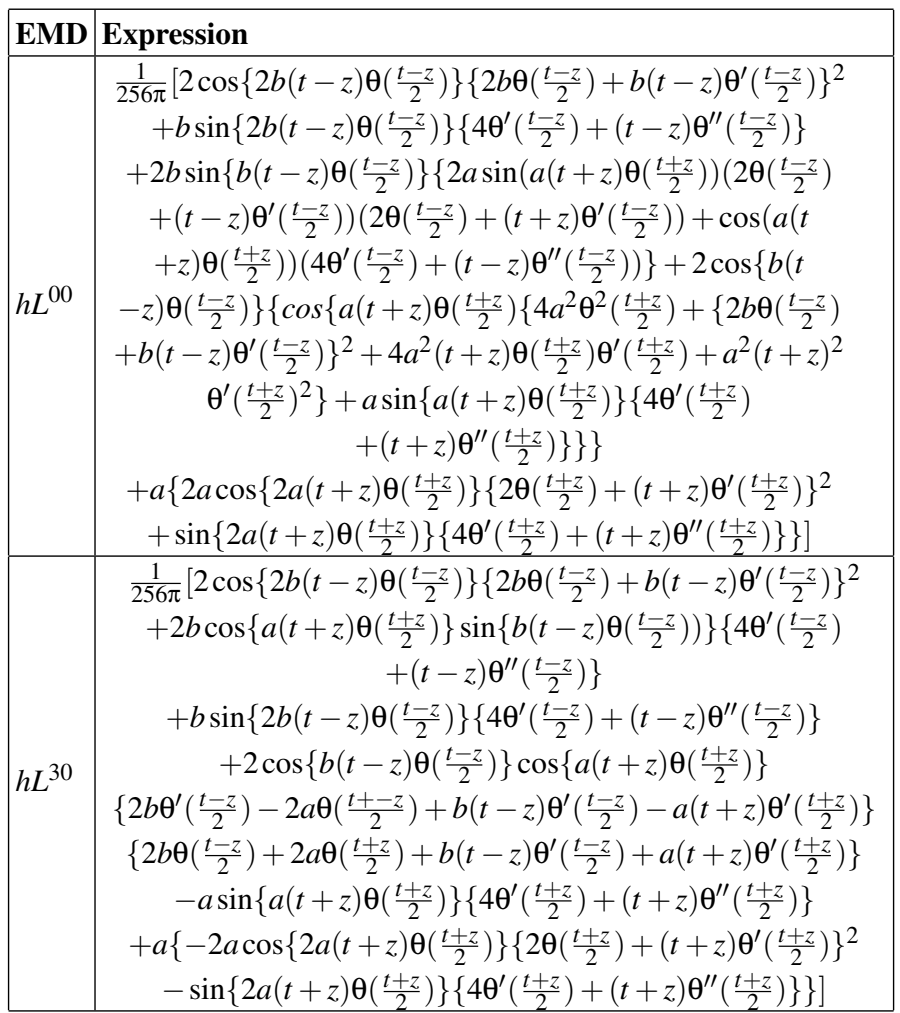

Table 1(c) Bell-Szekeres Metric: Bergmann's Prescription in GR and TPT \begin{tabular}{|l|l} 
EMD & Expression
\end{tabular}

\begin{tabular}{|c|c|}
\hline \multirow{3}{*}{$B^{00}$} & $\frac{1}{32 \pi}\left[\cos \left\{b(t-z) \theta\left(\frac{t-z}{2}\right)\right\}\left\{2 b \theta\left(\frac{t-z}{2}\right)+b(t-z) \theta^{\prime}\left(\frac{t-z}{2}\right)\right\}^{2}\right.$ \\
& $+b \sin \left\{b(t-z) \theta\left(\frac{t-z}{2}\right)\right\}\left\{4 \theta^{\prime}\left(\frac{t-z}{2}\right)+(t-z) \theta^{\prime \prime}\left(\frac{t-z}{2}\right)\right\}$ \\
& $+a\left\{a \cos \left\{a(t+z) \theta\left(\frac{t+z}{2}\right)\right\}\left\{2 \theta\left(\frac{t+z}{2}\right)+(t+z) \theta^{\prime}\left(\frac{t-z}{2}\right)\right\}^{2}\right.$ \\
& $\left.\left.+\sin \left\{a(t+z) \theta\left(\frac{t+z}{2}\right)\right\}\left\{4 \theta^{\prime}\left(\frac{t+z}{2}\right)+(t+z) \theta^{\prime \prime}\left(\frac{t+z}{2}\right)\right\}\right\}\right]$ \\
\hline \multirow{3}{*}{$B^{30}$} & $\frac{1}{32 \pi}\left[\cos \left\{b(t-z) \theta\left(\frac{t-z}{2}\right)\right\}\left\{2 b \theta\left(\frac{t-z}{2}\right)+b(t-z) \theta^{\prime}\left(\frac{t-z}{2}\right)\right\}^{2}\right.$ \\
& $+b \sin \left\{b(t-z) \theta\left(\frac{t-z}{2}\right)\right\}\left\{4 \theta^{\prime}\left(\frac{t-z}{2}\right)+(t-z) \theta^{\prime \prime}\left(\frac{t-z}{2}\right)\right\}$ \\
& $+a\left\{-a \cos \left\{a(t+z) \theta\left(\frac{t+z}{2}\right)\right\}\left\{2 \theta\left(\frac{t+z}{2}\right)+(t+z)\left\{\theta^{\prime}\left(\frac{t-z}{2}\right)\right\}^{2}\right.\right.$ \\
& $\left.\left.\left.-\sin \left\{a(t+z) \theta\left(\frac{t+z}{2}\right)\right\}\left\{4 \theta^{\prime}\left(\frac{t+z}{2}\right)+(t+z) \theta^{\prime \prime}\left(\frac{t+z}{2}\right)\right\}\right\}\right\}\right]$ \\
\hline
\end{tabular}

Table 1(d) Bell-Szekeres Metric: Möller's Prescription in GR and in TPT EMD Expression

\begin{tabular}{|c|c|}
\hline \multirow{4}{*}{$h B^{00}$} & $\frac{1}{32 \pi}\left[\cos \left\{b(t-z) \theta\left(\frac{t-z}{2}\right)\right\}\left\{2 b \theta\left(\frac{t-z}{2}\right)+b(t-z) \theta^{\prime}\left(\frac{t-z}{2}\right)\right\}^{2}\right.$ \\
& $+b \sin \left\{b(t-z) \theta\left(\frac{t-z}{2}\right)\right\}\left\{4 \theta^{\prime}\left(\frac{t-z}{2}\right)+(t-z) \theta^{\prime \prime}\left(\frac{t-z}{2}\right)\right\}$ \\
& $+a\left\{a \cos \left\{a(t+z) \theta\left(\frac{t+z}{2}\right)\right\}\left\{2 \theta\left(\frac{t+z}{2}\right)+(t+z) \theta^{\prime}\left(\frac{t-z}{2}\right)\right\}^{2}\right.$ \\
& $\left.\left.+\sin \left\{a(t+z) \theta\left(\frac{t+z}{2}\right)\right\}\left\{4 \theta^{\prime}\left(\frac{t+z}{2}\right)+(t+z) \theta^{\prime \prime}\left(\frac{t+z}{2}\right)\right\}\right\}\right]$ \\
\hline \multirow{4}{*}{$h B^{30}$} & $\frac{1}{32 \pi}\left[\cos \left\{b(t-z) \theta\left(\frac{t-z}{2}\right)\right\}\left\{2 b \theta\left(\frac{t-z}{2}\right)+b(t-z) \theta^{\prime}\left(\frac{t-z}{2}\right)\right\}^{2}\right.$ \\
& $+b \sin \left\{b(t-z) \theta\left(\frac{t-z}{2}\right)\right\}\left\{4 \theta^{\prime}\left(\frac{t-z}{2}\right)+(t-z) \theta^{\prime \prime}\left(\frac{t-z}{2}\right)\right\}$ \\
& $+a\left\{-a \cos \left\{a(t+z) \theta\left(\frac{t+z}{2}\right)\right\}\left\{2 \theta\left(\frac{t+z}{2}\right)+(t+z)\left\{\theta^{\prime}\left(\frac{t-z}{2}\right)\right\}^{2}\right.\right.$ \\
& $\left.\left.\left.-\sin \left\{a(t+z) \theta\left(\frac{t+z}{2}\right)\right\}\left\{4 \theta^{\prime}\left(\frac{t+z}{2}\right)+(t+z) \theta^{\prime \prime}\left(\frac{t+z}{2}\right)\right\}\right\}\right\}\right]$ \\
\hline
\end{tabular}

